\title{
The neurobiology of gustation in insect disease vectors: progress and potential
}

\author{
Richard Benton \\ Center for Integrative Genomics \\ Faculty of Biology and Medicine \\ Génopode Building \\ University of Lausanne \\ $\mathrm{CH}-1015$ \\ Lausanne \\ Switzerland
}

"Corresponding author:

T: ++41216923932

F: ++41216923965

E: Richard.Benton@unil.ch 


\section{Summary}

For insect vectors of human diseases, mealtimes are a key moment of infection. Understanding how and when such species decide on what to feed is both an interesting problem in sensory neurobiology and a source of information for intervention of these behaviors to control spread of infectious agents. Here I review the current knowledge of the molecular and cellular mechanisms of gustation in insect disease vectors, covering blood-feeders as well as scavengers that spread pathogens indirectly. I also consider how these behaviors are modulated over short and long timescales, and describe efforts to artificially modulate them. Though a relatively nascent field, gustatory neurobiology in insect vectors has much promise for future fundamental discoveries and practical applications.

\section{Introduction}

Transmission of pathogens from insect vectors to humans (or other animals) and vice versa - occurs most directly through bites by blood-feeding species such as mosquitoes [1], sand flies [2], fleas [3] and triatomine (or "kissing") bugs [4] (Table 1). Disease spread by insects can also be more indirect: blowflies and cockroaches, for example, may inadvertently inoculate our food with harmful microorganisms carried on or in their bodies [3] (Table 1). Understanding on what, when and how insect vectors feed is therefore not only of interest to chemical ecologists and sensory neurobiologists, but also has enormous potential for the development of intervention strategies to reduce disease transmission.

Investigations of food-seeking behaviors by insect vectors over the last century have revealed enormous diversity in feeding preferences [5-7]. Some hematophagous species are highly restricted in their source of blood meals: the body louse Pediculus humanus humanus is an obligate parasite that feeds only on humans [8]. A few mosquito subspecies are also highly specialized for human blood (e.g., Anopheles gambiae sensu stricto and Aedes aegypti aegypti), while others display a broader host range, such as Culex species, which are anthropophilic in the tropics but feed on birds and various mammals in other 
regions [1,9]. Regardless of the source, blood meals are essential for females of these species to produce eggs, likely due to their high protein content [5]. In nonhematophagous species, the propensity to spread pathogens is, for some, like blowflies, due to their feeding on microbe-rich carrion and dung; for others, such as cockroaches, it may be linked to their omnivorous diet and indiscriminate foraging. Feeding behaviors, particularly in hematophagous insects, are also highly plastic, such as the profound suppression of feeding exhibited by female mosquitoes for several days after a blood meal [1].

Despite the central role of gustatory behaviors for survival and reproduction of insect disease vectors, knowledge of the underlying neurobiology is still fragmentary, and lags some way behind that of odor-guided behaviors $[10,11]$. However, recent work, employing genomic, molecular, physiological, pharmacological and neurogenetic approaches has begun to provide insights into the molecular and neuronal basis of tasting and feeding in these species. In this review, I consider some of these advances, and discuss open questions and future priorities.

\section{Gustatory sensory organs and neurons: variations on common themes}

The sensory structures that insects use to assess non-volatile chemicals from food/host sources have been studied anatomically and functionally for decades in many different species, most famously reviewed in Dethier's monograph on the blowfly Phormia regina [6]. In the last 15 years, systematic investigations in the (non-vector) vinegar fly Drosophila melanogaster have extended the insights first obtained in other insects to provide a broad (though still incomplete) understanding of chemical detection and discrimination by the gustatory system [12]. Despite the enormous diversity of salient chemicals for different species, and overt morphological distinctions in insects' mouthparts [5], there are many commonalities in the properties of taste sensors: chemicals are detected by gustatory sensory neurons housed within cuticular hairs (sensilla), which decorate various appendages including the labellum of the proboscis (Figure 1a-b), legs, wing margins, and ovipositor; other sensilla line the pharyngeal lumen through which food passes prior to digestion [13,14]. In some species, such as the triatomine bug Rhodnius prolixus, sensilla on the antenna (an olfactory organ in 
most insects) also appear to contribute to non-volatile chemical detection [13]. Gustatory sensilla have a pore at their tip to allow movement of chemicals from the environment into the sensillar lymph fluid that bathes the ciliated endings of sensory dendrites. Individual sensilla house stereotyped clusters of neurons (typically 2-5), which exhibit physiological sensitivity to a specific class of appetitive (e.g., sugars, low salt concentrations, water) or aversive (e.g., bitter compound/high salt concentrations) stimuli $[6,12]$. These properties led to an initially simple model of taste coding where a limited number of broadly-tuned sensory channels (e.g., "sweet" and "bitter" neurons) are tightly coupled to acceptance or rejection behaviors.

Recent efforts in mosquitoes have started to examine this model in more detail through comparisons of responses to a panel of ecologically-relevant sugars. Within the labellar sensilla analyzed, "sweet" neurons were found to be most sensitive physiologically to sucrose and melezitose, as well as mixtures of fructose and glucose (but not these monosaccharides alone), with concomitant behavioral preference for - and survival of animals on - these sugars $[15,16]$. Such observations suggest the existence of refined mechanisms for perception and discrimination of these compounds, as is the case in D. melanogaster [17] and blowflies [6]. Bitter compound detection also appears to be complex in mosquitoes, involving activation of the "bitter neuron" and inhibition of the physiological sensitivity of both the "sweet" and water neurons $[15,18,19]$, as well as contributions from internal gustatory organs [20]. In triatomine bugs, behavioral studies reveal their capacity to discriminate different bitter tastants [21]. Moreover, these compounds can inhibit feeding behavior both prior to ingestion, through activation of antennal bitter neurons, and after their first mouthful, potentially through pharyngeal sensilla [13]. Thus, contrary to simple models, these observations imply that individual tastant classes act through multiple neural pathways.

It remains unclear whether acceptance and rejection of sweet and bitter tastants in blood-feeding insects is related to host choice rather than, for example, assessment of carbohydrate-rich plant nectar. There are anecdotal claims that humans with higher skin secretions of sugars are more attractive to mosquitoes, but such a link (if true) could be due to indirect influences, for example, the skin microbiota, which are a source of many odiferous molecules 
[22]. Intravital imaging of feeding in An. gambiae has revealed prolonged probing of skin by the proboscis both prior to and after penetration [23], suggesting that both superficial and intradermal cues are assessed. However, the relevant signals and sensory pathways are unclear, and receptors for known bloodderived feeding stimulants, such as ATP for Ae. aegypti [24], have not been identified. This might be because blood normally comes into contact only with internal gustatory sensory organs (as it is ingested), which are much harder to analyze by electrophysiological methods [20]. It is also possible that olfactory attractants, such as L-lactic acid [25], are sensed by the gustatory system. Finally, the contribution of chemosensory sensilla on other appendages, such as legs, wing margins $[26,27]$ and the ovipositor [28] to feeding or other behavioral decisions, is poorly understood.

\section{Divergent (and predominantly orphan) taste receptors}

Molecular receptors for taste stimuli are also starting to be characterized, building upon their initial identification in $D$. melanogaster [12]. These receptors comprise two large repertoires of presumed ligand-gated ion channels, the Gustatory Receptors (GRs) and lonotropic Receptors (IRs), as well as certain members of the Pickpocket and Transient Receptor Potential families [12]. Perireceptor gustatory-expressed "Odorant" Binding Proteins (OBPs) may also function in initial chemical recognition [29], but their role in sensory transduction remains unclear.

Within the increasing deluge of genomes and transcriptomes of insect disease vectors, homologous chemosensory receptor and OBP genes have been eagerly sought [30-37]. Comparative genomic and phylogenetic analyses with $D$. melanogaster receptors of known function have allowed predictions about roles of particular receptors, for example, of a clade of GRs that detect sugars [38]. Notably, these receptors appear to be lacking in tsetse flies [36], which may be related to the obligate hematophagous nature of both females and males in these species (contrasting with mosquitoes, where males feed on nectar). For many taste receptors, clear orthologous relationships have been difficult to define $[30,39]$, which probably reflects the evolution of distinct taste preferences of different species. 
Tissue-specific RNA-sequencing analyses in mosquitoes have also started to reveal in which chemosensory organs different receptor genes are expressed [40-43], although cellular level (co)expression properties have not yet been described. Furthermore, despite phylogenetically-guided predictions, soberingly few taste ligand/receptor relationships have been defined for GRs and IRs. This is in large part due to the inability to express these receptors in heterologous systems where they can be functionally interrogated. The lack of progress stands in contrast to the relative ease of deorphanization of Odorant Receptors (ORs) $[44,45]$, and implies that taste receptors function only in very specific (but stillundefined) combinations and/or require additional co-factors or a particular cellular environment. Recently, an antennal neuron population in $D$. melanogaster was proposed as a potential "GR decoder" system, in which heterologous expression of An. gambiae GR25a (an ortholog of the D. melanogaster fructose receptor GR43a [46]) was observed to confer sugar sensitivity [47]. However, it is unclear whether the ligand specificity of receptors heterologously expressed in this decoder always faithfully reflects their endogenous properties [48].

It is important to recognize that the anthropomorphic definition that distinguishes gustatory and olfactory pathways in mammals [49] is only partly relevant to insects. Although olfaction is mediated by neurons in the antennae and maxillary palps that project to the antennal lobe, the olfactory receptor repertoires (ORs and a subset of IRs) are molecularly related to the two main taste receptor families [50,51]. The strict segregation of taste and olfaction is further blurred in mosquitoes (and probably many other insects [52]): in $A n$. gambiae, neurons housed in the proboscis can respond to volatile chemicals (i.e., without direct contact with the stimulus source) [53] (although see [16]), and a subset of ORs is expressed in this organ in several mosquito species [42,53-56]. Expression of some Ae. aegypti ORs is also detected in the legs [42]. Intriguingly, ORs that can detect certain blood volatiles (amongst other non-blood chemicals) are found in the piercing/sucking stylet of Ae. aegypti [55]. However, it is unclear whether these receptors sense blood before or only after the stylet has penetrated the skin, and equivalent OR-expressing stylet neurons have not been observed in An. gambiae [57]. Finally, new transgenic neural tracing tools in $A n$. gambiae have revealed that OR-expressing neurons in the proboscis project to the primary taste center, the subesophageal zone (SEZ) [57], and not - as 
previously suggested [53] - the antennal lobe (Figure 1c). However, because of the paucity of knowledge of the roles of taste-organ expressed ORs in vivo, it remains unclear whether such anatomical projections underlie sensory integration of olfactory and gustatory stimuli in the SEZ or, perhaps more simply, that these ORs function essentially as taste receptors.

\section{Plasticity in feeding behaviors over multiple timescales}

As in all animals, the feeding behaviors of insect disease vectors are flexible, adapting on both short and long-timescales according to the internal state of the individual and the selection pressures in the environment. Beyond the basic scientific interest, understanding these processes is of substantial epidemiological significance, because of their influence on the temporal and spatial dynamics of pathogen transmission.

Mosquitoes provide a striking example of cyclical feeding behavior: in female Ae. aegypti, for example, following a blood meal, host-seeking and bloodfeeding are suppressed for three days, permitting egg production, fertilization and oviposition, after which a new round of host-seeking and blood-feeding begins [1]. The orchestration of this dramatic switch in feeding behavior was found, nearly 50 years ago, to depend upon a humoral substance [58]. Subsequently, Head Peptide-I (HP-I), a short neuropeptide F-related molecule, apparently present in the nervous system, was proposed as a key molecular component of this substance [59]. However, recent expression analyses did not detect HP-I in the brain (but rather in the male accessory gland, from where it might be transferred to females during copulation) [60]. Moreover, an in vitro pharmacologically defined neuropeptide Y-like receptor for HP-I, NPYLR1, is dispensable for any feeding-related behaviors [61]. Thus, the contribution and site of action of HP-I are unresolved.

While the precise determinant(s) of the dramatic behavioral switch are unknown, neurotranscriptomic analysis of female Ae. aegypti before and after a blood meal has revealed co-incident up- or down-regulation of many hundreds of genes [42]. (Gene expression changes pre- and post-blooding feeding have also been described in An. gambiae, in more limited analyses of olfactory organs $[62,63])$. These modulated genes include those encoding chemosensory 
receptors, neuropeptides and neurotransmitters, consistent with a substantial reweighting of gustatory and olfactory detection and processing capacities of these animals. However, the relationship of these transcriptional changes with behavioral phenotypes remains to be determined.

Shorter-term modulation of gustatory-dependent behaviors in both sexes is likely to be prevalent (e.g., circadian control [64]). In addition, observations of non-associative and associative learning of taste stimuli in mosquitoes [65,66] and triatomine bugs $[13,21]$ indicate that these animals also rely on prior experience to guide host choice. The underlying molecular mechanisms are largely obscure, but probably involve conserved neurotransmitter and neuropeptide signals, such as serotonin [67] and allostatins [68], acting in central circuits. Recent studies also hint at gustatory sensory neurons being a direct target for neuromodulation: in Ae. aegypti, a receptor for kinin-family neuropeptides (which have multifunctional roles in the alimentary canal) was found to be expressed in labellar and leg taste neurons, and pharmacological treatment (through topical application or feeding) of non-degradable kinin analogs suppressed sugar detection and feeding [69]. The natural context for the action of this peripheral neuropeptide signal is unclear, but these observations are consistent with evidence from $D$. melanogaster that sensory neurons are an important target for pathways modulating taste perception and behavior [12].

Beyond endogenous control mechanisms, one intriguing way in which disease vector feeding (amongst other behaviors) might be modulated is by the infective agent itself. For example, in sandflies (Lutzomyia longipalpis), the protozoan parasite Leishmania causes the insect to persist with blood feeding and to bite multiple mammalian hosts [70]. In mosquitoes, diverse influences of the parasite have been reported [71]. In some cases, infection is simply detrimental to the insect host (and parasite): Culex pipiens' starvation resistance is compromised by the presence of Plasmodium [72]. In Anopheles mosquitoes, the presence of the infectious sporozoites (but not the non-infectious oocysts) can increase female probing and biting persistence, while decreasing blood meal size (which may lead to multiple feeds on different host individuals) [71]. While it is appealing to credit the parasite with devious manipulative abilities, it is unclear to what extent these effects are truly adaptive (representing "extended phenotypes"); complementary field studies are required to determine the 
significance of the observations made in controlled laboratory assays to the efficiency of parasite transmission in nature. Moreover, some changes in mosquito feeding can also be induced with heat-killed E. coli, potentially linked to alterations in gut insulin signaling [73-75]. These observations raise the possibility that many of the consequences of parasite infection - though potentially beneficial for the parasite - may simply be the by-product of a general immune response to microbial infection.

Insects can also exhibit plasticity over evolutionary time scales - facilitated by their relatively large population size and short generation time - which allows them to adapt their behavior to novel selective pressures in the environment. Peripheral chemosensory systems display particularly strong genetic signatures of selection over short timescales in insects [76]. In the olfactory system, the evolution of preference of Ae. aegypti strains to selectively target humans is linked to increases in the expression and sensitivity of an OR for sulcatone, a particularly abundant component of human odor [77] (although mosquito behavioral responses to this volatile appear complex [78]). In the gustatory system, the species-specificity of GRs and IRs strongly implies adaptation of taste preferences to particular ecological niches [30,39], but the dearth of knowledge about specific ligand/receptor relationships (as well as limited knowledge of the chemical ecology of species-specific taste stimuli) precludes further insight.

One fascinating example of sensory evolution at the neuronal level in the gustatory system was discovered in the cockroach Blattella germanica. Within natural populations, "glucose-averse" strains have been identified, which exhibit physiological sensitivity to glucose (but not other sugars) in the canonical bittersensing taste neuron $[79,80]$. The molecular basis for this physiological trait is unknown, but is thought to reflect a broadening of tuning of bitter-neuron receptors, rather than "ectopic" expression of sugar-sensing GRs in these cells [79]. Although this trait comes at fitness cost - due to impaired nutritional intake [81] - this unusual sensory coding mechanism enables cockroaches to avoid human-laid toxic baits in which glucose is used as a primary phagostimulant. Recent anthropogenically-imposed selection pressures may therefore have driven the global spread of glucose-averse cockroach populations [82]. 


\section{Manipulation of feeding: desired and undesired effects}

One motivation to study the chemosensory biology of insect disease vectors is to allow the development of rational strategies to manipulates host feeding and disease spread. As introduced above, attractive sugar toxic baits (ASTBs) represent a common approach [83], which relies on most vectors' innate feeding on sweet substances to induce their consumption of toxins. Beyond poisoning insects, the most widely used chemical manipulator of their behavior is $N, N$ Diethyl-meta-toluamide (DEET), which was developed in a systematic effort more than 50 years ago; many others have been discovered serendipitously $[84,85]$. For any of these compounds, their mechanism of action - and even whether they all act as "repellents" - remains incompletely understood, but it appears that the most effective, such as DEET, operate on multiple sensory pathways $[84,85]$. DEET has important effects in the vapor phase, but precisely how it influences the olfactory system is still-debated [84-88]. Soon after its initial development, however, DEET was also shown to be a direct blood-feeding deterrent for Ae. aegypti [89]; this finding was later generalized to other commonly-used "repellents" and different mosquito species [90], as well as to sugar-feeding behavior in $D$. melanogaster [91]. Electrophysiological analyses revealed DEET (and certain other repellents) could activate bitter-sensing labellar neurons in diverse mosquitoes and in $D$. melanogaster [18,19,91,92]. Moreover, genetic studies in $D$. melanogaster demonstrated that the physiological and behavioral responses to DEET in the gustatory system depend, in part, upon specific GRs [91]; how these receptors are targeted by this chemical is unknown. These observations support a model in which DEET (and perhaps other repellents) act by stimulating aversive gustatory pathways that counteract the activity of appetitive gustatory channels. However, it is unclear how easily information on the impact of DEET on sugar-feeding behavior in $D$. melanogaster can be extrapolated to mosquitoes, where skin landing and blood consumption are likely to involve a more complex set of positive gustatory cues and chemosensory appendages.

While a better understanding of taste-evoked behaviors in disease vectors

might enable development of more effective, selective, and safer repellents, artificial application of chemicals can of course have unanticipated consequences 
in nature. In some cases, as in cockroaches' response to toxic baits described above, species may adapt and subvert our efforts [79]. In other cases, our interventions cause serious ecological damage, as is the case for the neonicotinoid family of insecticides, whose widespread use has been linked to devastating impact on important pollinators such as honey bees [93]. Unexpectedly, sublethal doses of neonicotinoids (in sugar solutions) promote feeding preferences by bees [94], even though their consumption ultimately disrupts learning, navigation and motor function. The preference does not appear to be due to sensory detection of these compounds [94], suggesting a central site of action of neonicotinoids. Importantly, this implies that bees (unlike cockroaches' avoidance of poisoned baits) may be unable to rely on peripheral gustatory mechanisms to avoid ingesting these toxic chemicals.

\section{Conclusions}

Despite the broad appreciation of gustatory-related behaviors in diverse insect vectors, our current knowledge of the underlying neurobiology remains quite superficial, and - as illustrated here - often dispersed across many different species. Key future priorities should include comprehensive analyses of the physiological responses of gustatory neurons and the in situ expression patterns of candidate taste receptors, as well as the development of heterologous systems for receptor deorphanization. Comparative genomic and functional data across closely-related species with distinct feeding preferences should help researchers home in on important sensory pathways. Moreover, identification of receptors for ecologically important phagostimulants will provide us with targets for potential pharmacological manipulation in nature.

Encouragingly, the establishment of genetic tools to visualize and manipulate neural circuits in several disease vectors - notably mosquitoes - now makes it conceivable to derive causal relationship between gustatory cues, neural representations and behaviors. Genetic approaches will also be essential to cleanly dissect the relative contributions of gustatory, olfactory and other sensory inputs to feeding behaviors, as well as for interrogating the poorly understood neuromodulatory mechanisms by which internal state and experience influence them. 
Finally, it will be interesting to expand these investigations to non-volatile chemical signals that control social interactions (e.g., cuticular hydrocarbons [95], which function as sex pheromones in drosophilids [96]) or oviposition site selection $[97,98]$. Such behaviors are equally important for the success - and so devastating impact - of insect disease vectors. 


\section{Acknowledgements}

I am grateful to Patrick Guerin, Sébastien Kessler, Christopher Potter and Olena Riabinina for sharing images. I thank Thomas Auer, Emily Dennis, Patrick Guerin, Sébastien Kessler, Frédéric Marion-Poll, Christopher Potter, Lucia Prieto-Godino and Juan Sanchez-Alcaniz for discussions and/or comments on the manuscript. I apologize for colleagues for being unable to cite all relevant studies due to space constraints. Research in my laboratory is supported by the University of Lausanne, an ERC Consolidator Grant (615094) and the Swiss National Science Foundation.

\section{References}

1. Clements AN: The Biology of Mosquitoes: Cabi; 1992.

2. Ready PD: Biology of phlebotomine sand flies as vectors of disease agents. Annu Rev Entomol 2013, 58:227-250.

3. Rodhain F: Insects as vectors: systematics and biology. Rev Sci Tech 2015, 34:83-96, 67-82.

4. Barrozo RB, Reisenman CE, Guerenstein P, Lazzari CR, Lorenzo MG: An inside look at the sensory biology of triatomines. J Insect Physiol 2016.

5. Lehane MJ: The biology of blood-sucking in insects edn 2nd ed. Cambridge: Cambridge University Press; 2005.

6. Dethier VG: The Hungry Fly. Cambridge: Harvard University Press; 1976.

7. Mullins DE: Physiology of Environmental Adaptations and Resource Acquisition in Cockroaches. Annual Review of Entomology, Vol 60 2015, 60:473-492. 
8. Raoult $\mathrm{D}$, Roux $\mathrm{V}$ : The body louse as a vector of reemerging human diseases. Clinical Infectious Diseases 1999, 29:888-911.

9. Takken W, Verhulst NO: Host preferences of blood-feeding mosquitoes. Annu Rev Entomol 2013, 58:433-453.

10. McBride CS: Genes and Odors Underlying the Recent Evolution of Mosquito Preference for Humans. Curr Biol 2016, 26:R41-R46.

11. Reisenman CE, Lei H, Guerenstein PG: Neuroethology of OlfactoryGuided Behavior and Its Potential Application in the Control of Harmful Insects. Front Physiol 2016, 7:271.

12. Freeman EG, Dahanukar A: Molecular neurobiology of Drosophila taste. Curr Opin Neurobiol 2015, 34:140-148.

- 13. Pontes G, Minoli S, Insaurralde IO, de Brito Sanchez MG, Barrozo RB: Bitter stimuli modulate the feeding decision of a blood-sucking insect via two sensory inputs. Journal of Experimental Biology 2014, 217:37083717.

A simple but elegant analysis of triatomine bug ( $R$. prolixus) feeding behavior, which shows that bitter stimuli deter feeding both through external antennal gustatory sensilla and, possibly, internal sensory structures that contact food after ingestion.

14. Ledue EE, Chen YC, Jung AY, Dahanukar A, Gordon MD: Pharyngeal sense organs drive robust sugar consumption in Drosophila. Nature Communications 2015, 6.

15. Kessler S, Vlimant M, Guerin PM: The sugar meal of the African malaria mosquito Anopheles gambiae and how deterrent compounds 
interfere with it: a behavioural and neurophysiological study. Journal of Experimental Biology 2013, 216:1292-1306.

- 16. Kessler S, Vlimant M, Guerin PM: Sugar-sensitive neurone responses and sugar feeding preferences influence lifespan and biting behaviours of the Afrotropical malaria mosquito, Anopheles gambiae. J Comp Physiol A Neuroethol Sens Neural Behav Physiol 2015, 201:317329.

A systematic physiological analysis of sugar-sensing neurons in An. gambiae, which correlates their tuning properties with mosquitoes' behavioral preferences.

17. Fujii S, Yavuz A, Slone J, Jagge C, Song X, Amrein H: Drosophila Sugar Receptors in Sweet Taste Perception, Olfaction, and Internal Nutrient Sensing. Curr Biol 2015, 25:621-627.

18. Sparks JT, Dickens JC: Bitter-sensitive gustatory receptor neuron responds to chemically diverse insect repellents in the common malaria mosquito Anopheles quadrimaculatus. Naturwissenschaften 2016, 103:39.

19. Sparks JT, Dickens JC: Electrophysiological Responses of Gustatory Receptor Neurons on the Labella of the Common Malaria Mosquito, Anopheles quadrimaculatus (Diptera: Culicidae). J Med Entomol 2016.

20. Kessler S, Gonzalez J, Vlimant M, Glauser G, Guerin PM: Quinine and artesunate inhibit feeding in the African malaria mosquito Anopheles gambiae: the role of gustatory organs within the mouthparts. Physiological Entomology 2014, 39:172-182. 
21. Asparch Y, Pontes G, Masagué S, Minoli S, Barrozo RB: Kissing bugs can generalize and discriminate between different bitter compounds. $J$ Physiol 2016.

22. Verhulst NO, Takken W, Dicke M, Schraa G, Smallegange RC: Chemical ecology of interactions between human skin microbiota and mosquitoes. Fems Microbiology Ecology 2010, 74:1-9.

23. Choumet V, Attout T, Chartier L, Khun H, Sautereau J, Robbe-Vincent A, Brey $P$, Huerre $M$, Bain $O$ : Visualizing non infectious and infectious Anopheles gambiae blood feedings in naive and saliva-immunized mice. PLoS One 2012, 7:e50464.

24. Galun R, Avidor Y, Barzeev M: Feeding Response in Aedes Aegypti Stimulation by Adenosine Triphosphate. Science 1963, 142:1674-+.

25. Dekker T, Steib B, Carde RT, Geier M: L-lactic acid: a human-signifying host cue for the anthropophilic mosquito Anopheles gambiae. Medical and Veterinary Entomology 2002, 16:91-98.

26. Raad H, Ferveur JF, Ledger N, Capovilla M, Robichon A: Functional Gustatory Role of Chemoreceptors in Drosophila Wings. Cell Rep 2016, 15:1442-1454.

27. Yanagawa A, Guigue AMA, Marion-Poll F: Hygienic grooming is induced by contact chemicals in Drosophila melanogaster. Frontiers in Behavioral Neuroscience 2014, 8.

28. van Lenteren JC, Ruschioni S, Romani R, van Loon JJA, Qiu YT, Smid HM, Isidoro N, Bin F: Structure and electrophysiological responses of gustatory organs on the ovipositor of the parasitoid Leptopilina heterotoma. Arthropod Structure \& Development 2007, 36:271-276. 
29. Jeong YT, Shim J, Oh SR, Yoon HI, Kim CH, Moon SJ, Montell C: An odorant-binding protein required for suppression of sweet taste by bitter chemicals. Neuron 2013, 79:725-737.

30. Kent LB, Walden KK, Robertson HM: The Gr family of candidate gustatory and olfactory receptors in the yellow-fever mosquito Aedes aegypti. Chem Senses 2008, 33:79-93.

31. Hill CA, Fox AN, Pitts RJ, Kent LB, Tan PL, Chrystal MA, Cravchik A, Collins $\mathrm{FH}$, Robertson HM, Zwiebel LJ: G protein-coupled receptors in Anopheles gambiae. Science 2002, 298:176-178.

32. Zhou X, Qian K, Tong Y, Zhu JJ, Qiu X, Zeng X: De novo transcriptome of the Hemimetabolous German cockroach (Blattella germanica). PLoS One 2014, 9:e106932.

- 33. Neafsey DE, Waterhouse RM, Abai MR, Aganezov SS, Alekseyev MA, Allen JE, Amon J, Arca B, Arensburger P, Artemov G, et al.: Mosquito genomics. Highly evolvable malaria vectors: the genomes of 16 Anopheles mosquitoes. Science 2015, 347:1258522.

Comparative genomic analyses of 16 anopheline species provide a wealth of insights into the biology of these disease vectors. Interestingly, chemosensory gene repertoires appear to diversify by changes in protein sequences rather than gene turnover.

34. Mesquita RD, Vionette-Amaral RJ, Lowenberger C, Rivera-Pomar R, Monteiro FA, Minx P, Spieth J, Carvalho AB, Panzera F, Lawson D, et al.: Genome of Rhodnius prolixus, an insect vector of Chagas disease, reveals unique adaptations to hematophagy and parasite infection. Proc Natl Acad Sci U S A 2015, 112:14936-14941. 
- 35. International Glossina Genome Initiative: Genome sequence of the tsetse fly (Glossina morsitans): vector of African trypanosomiasis. Science 2014, 344:380-386.

- 36. Macharia R, Mireji P, Murungi E, Murilla G, Christoffels A, Aksoy S, Masiga D: Genome-Wide Comparative Analysis of Chemosensory Gene Families in Five Tsetse Fly Species. Plos Neglected Tropical Diseases 2016, 10.

These two papers report genome analyses of multiple tsetse fly species, including comparisons of insect chemosensory receptor repertories, which reveal the absence of the conserved sugar-sensing GRs.

37. Andere AA, li RNP, Ray DA, Picard CJ: Genome sequence of Phormia regina Meigen (Diptera: Calliphoridae): implications for medical, veterinary and forensic research. BMC Genomics 2016, 17.

38. Kent LB, Robertson HM: Evolution of the sugar receptors in insects. $B M C$ Evol Biol 2009, 9:41.

39. Croset V, Rytz R, Cummins SF, Budd A, Brawand D, Kaessmann H, Gibson TJ, Benton R: Ancient protostome origin of chemosensory ionotropic glutamate receptors and the evolution of insect taste and olfaction. PLoS Genet 2010, 6:e1001064.

40. Sparks JT, Vinyard BT, Dickens JC: Gustatory receptor expression in the labella and tarsi of Aedes aegypti. Insect Biochem Mol Biol 2013, 43:1161-1171.

41. Sparks JT, Bohbot JD, Dickens JC: The genetics of chemoreception in the labella and tarsi of Aedes aegypti. Insect Biochem Mol Biol 2014, 48:816. 
- 42. Matthews BJ, McBride CS, DeGennaro M, Despo O, Vosshall LB: The neurotranscriptome of the Aedes aegypti mosquito. BMC Genomics 2016, 17:32.

A comprehensive deep RNA-sequencing analysis of multiple peripheral sensory tissues and the brain of Ae. aegypti males and pre/post-blood-fed females, which allows identification of tissue-specific, sex-specific, and feeding state-dependent neural gene expression.

43. Pitts RJ, Rinker DC, Jones PL, Rokas A, Zwiebel LJ: Transcriptome profiling of chemosensory appendages in the malaria vector Anopheles gambiae reveals tissue- and sex-specific signatures of odor coding. BMC Genomics 2011, 12:271.

44. Carey AF, Wang G, Su CY, Zwiebel LJ, Carlson JR: Odorant reception in the malaria mosquito Anopheles gambiae. Nature 2010, 464:66-71.

45. Wang G, Carey AF, Carlson JR, Zwiebel LJ: Molecular basis of odor coding in the malaria vector mosquito Anopheles gambiae. Proc Natl Acad Sci U S A 2010.

46. Miyamoto $\mathrm{T}$, Slone $\mathrm{J}$, Song $\mathrm{X}$, Amrein $\mathrm{H}$ : A fructose receptor functions as a nutrient sensor in the Drosophila brain. Cell 2012, 151:1113-1125.

47. Freeman EG, Wisotsky Z, Dahanukar A: Detection of sweet tastants by a conserved group of insect gustatory receptors. Proceedings of the National Academy of Sciences of the United States of America 2014, 111:1598-1603.

48. Amrein $\mathrm{H}$ : An expression system for Gustatory receptors - and why it failed. Fly (Austin) 2014, 8:232-233. 
49. Wyatt TD: Pheromones and Animal Behaviour: Communication by Smell and Taste. Oxford: Oxford University Press; 2003.

50. Vosshall LB, Stocker RF: Molecular Architecture of Smell and Taste in Drosophila. Annu Rev Neurosci 2007, 30:505-533.

51. Rytz R, Croset V, Benton R: lonotropic Receptors (IRs): Chemosensory ionotropic glutamate receptors in Drosophila and beyond. Insect Biochem Mol Biol 2013, 43:888-897.

52. Dippel S, Kollmann M, Oberhofer G, Montino A, Knoll C, Krala M, Rexer KH, Frank S, Kumpf R, Schachtner J, et al.: Morphological and Transcriptomic Analysis of a Beetle Chemosensory System Reveals a Gnathal Olfactory Center. BMC Biol 2016, 14:90.

53. Kwon HW, Lu T, Rutzler M, Zwiebel LJ: Olfactory responses in a gustatory organ of the malaria vector mosquito Anopheles gambiae. Proc Natl Acad Sci U S A 2006, 103:13526-13531.

54. Pitts RJ, Fox AN, Zwiebel LJ: A highly conserved candidate chemoreceptor expressed in both olfactory and gustatory tissues in the malaria vector Anopheles gambiae. Proc Natl Acad Sci U S A 2004, 101:5058-5063.

- 55. Jung JW, Baeck SJ, Perumalsamy H, Hansson BS, Ahn YJ, Kwon HW: A novel olfactory pathway is essential for fast and efficient bloodfeeding in mosquitoes. Sci Rep 2015, 5:13444.

Identification of two ORs expressed in the Ae. aegypti stylet; these receptors respond to blood-derived volatiles (and other compounds), and might contribute to blood-feeding behavior of this mosquito. 
56. Choo YM, Buss GK, Tan K, Leal WS: Multitasking roles of mosquito labrum in oviposition and blood feeding. Front Physiol 2015, 6:306.

- 57. Riabinina O, Task D, Marr E, Lin CC, Alford R, O'Brochta DA, Potter CJ: Organization of olfactory centres in the malaria mosquito Anopheles gambiae. Nat Commun 2016, 7:13010.

Transgenesis of An. gambiae with the powerful binary expression Q-system. Here, this system is used to analyze the peripheral and central organization of OR co-receptor (ORCO)-expressing neurons, revealing functional and/or anatomical overlap of olfactory and gustatory pathways.

58. Klowden MJ, Lea AO: Humoral Inhibition of Host-Seeking in Aedes Aegypti during Oocyte Maturation. Journal of Insect Physiology 1979, 25:231-235.

59. Matsumoto S, Brown MR, Crim JW, Vigna SR, Lea AO: Isolation and Primary Structure of Neuropeptides from the Mosquito, AedesAegypti, Immunoreactive to Fmrfamide Antiserum. Insect Biochemistry 1989, 19:277-283.

60. Predel R, Neupert S, Garczynski SF, Crim JW, Brown MR, Russell WK, Kahnt J, Russell DH, Nachman RJ: Neuropeptidomics of the Mosquito Aedes aegypti. Journal of Proteome Research 2010, 9:2006-2015.

- 61. Liesch J, Bellani LL, Vosshall LB: Functional and genetic characterization of neuropeptide Y-like receptors in Aedes aegypti. PLoS Negl Trop Dis 2013, 7:e2486.

One of the first loss-of-function genetics studies in Ae. aegypti, which cleanly shows that a pharmacologically-identified Neuropeptide Y-related receptor for Head Peptide-I is dispensable for host-seeking inhibition after blood-feeding; additional/other receptors and/or neuropeptides must be involved in this dramatic 
behavioral switch.

62. Rinker DC, Pitts RJ, Zhou X, Suh E, Rokas A, Zwiebel LJ: Blood mealinduced changes to antennal transcriptome profiles reveal shifts in odor sensitivities in Anopheles gambiae. Proc Natl Acad Sci $U S A$ 2013, 110:8260-8265.

63. Fox AN, Pitts RJ, Robertson HM, Carlson JR, Zwiebel LJ: Candidate odorant receptors from the malaria vector mosquito Anopheles gambiae and evidence of down-regulation in response to blood feeding. Proc Natl Acad Sci U S A 2001, 98:14693-14697.

64. Meireles ACA, Kyriacou CP: Circadian rhythms in insect disease vectors. Memorias Do Instituto Oswaldo Cruz 2013, 108:48-58.

65. Bernáth B, Antstett V, Guerin PM: Anopheles gambiae females readily learn to associate complex visual cues with the quality of sugar sources. J Insect Physiol 2016, 95:8-16.

66. Alonso WJ, Schuck-Paim C: The 'ghosts' that pester studies on learning in mosquitoes: guidelines to chase them off. Medical and Veterinary Entomology 2006, 20:157-165.

67. Siju KP, Hansson BS, Ignell R: Immunocytochemical localization of serotonin in the central and peripheral chemosensory system of mosquitoes. Arthropod Structure \& Development 2008, 37:248-259.

68. Zandawala M, Orchard I: Post-feeding physiology in Rhodnius prolixus: The possible role of FGLamide-related allatostatins. General and Comparative Endocrinology 2013, 194:311-317.

69. Kwon H, Ali Agha M, Smith RC, Nachman RJ, Marion-Poll F, Pietrantonio PV: Leucokinin mimetic elicits aversive behavior in mosquito Aedes 
aegypti (L.) and inhibits the sugar taste neuron. Proc Natl Acad Sci U S A 2016, 113:6880-6885.

70. Rogers ME, Bates PA: Leishmania manipulation of sand fly feeding behavior results in enhanced transmission. PLoS Pathog 2007, 3:e91.

71. Cator LJ, Lynch PA, Read AF, Thomas MB: Do malaria parasites manipulate mosquitoes? Trends in Parasitology 2012, 28:466-470.

72. Lalubin $F$, Deledevant $A$, Glaizot $O$, Christe $P$ : Natural malaria infection reduces starvation resistance of nutritionally stressed mosquitoes. Journal of Animal Ecology 2014, 83:850-857.

73. Cator LJ, George J, Blanford S, Murdock CC, Baker TC, Read AF, Thomas MB: 'Manipulation' without the parasite: altered feeding behaviour of mosquitoes is not dependent on infection with malaria parasites. Proceedings of the Royal Society B-Biological Sciences 2013, 280.

74. Cator LJ, Pietri JE, Murdock CC, Ohm JR, Lewis EE, Read AF, Luckhart S, Thomas MB: Immune response and insulin signalling alter mosquito feeding behaviour to enhance malaria transmission potential. Scientific Reports 2015, 5.

75. Ohm JR, Teeple J, Nelson WA, Thomas MB, Read AF, Cator LJ: Fitness consequences of altered feeding behavior in immune-challenged mosquitoes. Parasites \& Vectors 2016, 9.

76. Arguello JR, Cardoso-Moreira M, Grenier JK, Gottipati S, Clark AG, Benton R: Extensive local adaptation within the chemosensory system following Drosophila melanogaster's global expansion. Nature Commun 2016, 7:ncomms11855. 
- 77. McBride CS, Baier F, Omondi AB, Spitzer SA, Lutomiah J, Sang R, Ignell R, Vosshall LB: Evolution of mosquito preference for humans linked to an odorant receptor. Nature 2014, 515:222-227.

Evidence linking the increased expression and sensitivity of an Ae. aegypti OR for sulcatone (an odor emitted at high levels in humans compared to other mammals) to the evolution of behavioral preference of domesticated forms of this mosquito towards human hosts.

78. Logan JG, Birkett MA, Clark SJ, Powers S, Seal NJ, Wadhams LJ, Mordue AJ, Pickett JA: Identification of human-derived volatile chemicals that interfere with attraction of Aedes aegypti mosquitoes. Journal of Chemical Ecology 2008, 34:308-322.

- 79. Wada-Katsumata A, Silverman J, Schal C: Changes in taste neurons support the emergence of an adaptive behavior in cockroaches. Science 2013, 340:972-975.

This study describes the exhibition of sensitivity to D-glucose by "bitter-sensing" gustatory neurons in multiple wild-caught strains of cockroaches, suggesting a mechanism by which they can avoid human-laid toxic baits containing this normally-phagostimulant sugar.

80. Wada-Katsumata A, Silverman J, Schal C: Differential Inputs from Chemosensory Appendages Mediate Feeding Responses to Glucose in Wild-Type and Glucose-Averse German Cockroaches, Blattella germanica. Chemical Senses 2011, 36:589-600.

81. Jensen K, Schal C, Silverman J: Suboptimal nutrient balancing despite dietary choice in glucose-averse German cockroaches, Blattella germanica. Journal of Insect Physiology 2015, 81:42-47. 
82. Shik JZ, Schal C, Silverman J: Diet specialization in an extreme omnivore: nutritional regulation in glucose-averse German cockroaches. Journal of Evolutionary Biology 2014, 27:2096-2105.

83. Faraji A, Unlu I: The Eye of the Tiger, the Thrill of the Fight: Effective Larval and Adult Control Measures Against the Asian Tiger Mosquito, Aedes albopictus (Diptera: Culicidae), in North America. J Med Entomol 2016, 53:1029-1047.

84. Leal W: The enigmatic reception of DEET - the gold standard of insect repellents. Current Opinion in Insect Science 2014, 6:93-98.

85. DeGennaro M: The mysterious multi-modal repellency of DEET. Fly (Austin) 2015, 9:45-51.

86. Silbering AF, Bell R, Munch D, Cruchet S, Gomez-Diaz C, Laudes T, Galizia CG, Benton R: Ir40a neurons are not DEET detectors. Nature 2016, 534:E5-7.

87. Xu P, Choo YM, De La Rosa A, Leal WS: Mosquito odorant receptor for DEET and methyl jasmonate. Proc Natl Acad Sci U S A 2014, 111:16592-16597.

88. Syed Z, Leal WS: Mosquitoes smell and avoid the insect repellent DEET. Proceedings of the National Academy of Sciences of the United States of America 2008, 105:13598-13603.

89. Bar-Zeev M, Schmidt C: Action of a Repellent as Indicated by a Radioactive Tracer. Journal of Economic Entomology 1959, 52:268-269.

90. Klun JA, Khrimian A, Debboun M: Repellent and deterrent effects of SS220, Picaridin, and Deet suppress human blood feeding by Aedes 
aegypti, Anopheles stephensi, and Phlebotomus papatasi. J Med Entomol 2006, 43:34-39.

91. Lee $\mathrm{Y}, \mathrm{Kim} \mathrm{SH}$, Montell $\mathrm{C}$ : Avoiding DEET through insect gustatory receptors. Neuron 2010, 67:555-561.

92. Sanford JL, Shields VD, Dickens JC: Gustatory receptor neuron responds to DEET and other insect repellents in the yellow-fever mosquito, Aedes aegypti. Naturwissenschaften 2013, 100:269-273.

93. Decourtye A, Devillers J: Ecotoxicity of neonicotinoid insecticides to bees. Adv Exp Med Biol 2010, 683:85-95.

- 94. Kessler SC, Tiedeken EJ, Simcock KL, Derveau S, Mitchell J, Softley S, Radcliffe A, Stout JC, Wright GA: Bees prefer foods containing neonicotinoid pesticides. Nature 2015, 521:74-76.

A cautionary study reporting the preference of bees for foods containing neonicotinoid insecticides, despite multiple adverse effects of these chemicals on their behavior. This preference does not appear to be due to gustatory detection of these insecticides, suggesting that these chemicals exert influence on the central nervous system.

95. Vaníčková L, Canale A, Benelli G: Sexual chemoecology of mosquitoes (Diptera, Culicidae): Current knowledge and implications for vector control programs. Parasitology International 2016, S1383-5769:3005830057.

96. Auer T, Benton R: Sexual circuitry in Drosophila. Current Opinion in Neurobiology 2016, 38:18-26. 
97. Suh E, Choe DH, Saveer AM, Zwiebel LJ: Suboptimal Larval Habitats Modulate Oviposition of the Malaria Vector Mosquito Anopheles coluzzii. Plos One 2016, 11.

98. Afify A, Galizia CG: Chemosensory Cues for Mosquito Oviposition Site Selection. Journal of Medical Entomology 2015, 52:120-130. 


\section{Legends}

\section{Table 1. Insect disease vectors}

A non-comprehensive overview of the insect vectors discussed in this article. "Biological vectors" are those in which the infectious agent must spend part of its life cycle within the insect host; "mechanical vectors" are passive carriers of the infectious agent [3]. Host ranges may vary within species (e.g., An. gambiae and Ae. aegypti; see text). Genus names are given for bacteria where multiple species may be pathogenic.

Figure 1. Chemosensory organs and neurons in Anopheles gambiae

(a) Light sheet fluorescence microscopy image of the head of a female An. gambiae showing the main chemosensory organs. Some chemosensory neurons (in both olfactory and gustatory appendages) are transgenically labeled with GFP (green) using the Q-system (genotype: Orco-QF2,QUAS-mCD8:GFP) [57]. Image kindly provided by Olena Riabinina and Christopher Potter (Johns Hopkins University School of Medicine).

(b) Scanning electron micrograph showing a dorsal view of the extremity of the proboscis of a female An. gambiae. Scale bar, $50 \mu \mathrm{m}$. Reproduced from [15] under a Creative Commons license.

(c) Top: Confocal image of a whole mount brain of a female An. gambiae (OrcoQF2, QUAS-mCD8:GFP) - stained with anti-GFP (green) and the neuropil marker nc82 (magenta) - showing the projection of these neurons to the primary olfactory center (antennal lobe; neurons from the antennae and maxillary palps) and the gustatory center (subesophageal zone; neurons from the proboscis). Higher magnification images of the chemosensory centers are shown below. Reproduced from [57] under a Creative Commons license. 


\section{Table 1. Insect disease vectors}

Species Common name Vector type Blood meal source Parasite (disease)

Anopheles gambiae malaria mosquito Biological mammals, birds Plasmodium (malaria)

yellow fever mosquito Biological mammals, birds

Plasmodium (malaria)

aegyp

yellow fever mosquito Biological mammals, birds

Culex pipiens

common house mosquito Biological birds, mammals

sandfly

Biological mammals

Rhodnius prolixus triatomine/kissing bug Biological mammals, birds

Blattella germanic

cockroach Mechanical N/A

West Nile virus (West Nile fever); Japanese encephalitis virus (Japanese encephalitis), Zika virus (Zika fever, microcephaly)

Leishmania (Leishmaniasis)

Rettsia prowazekii (epidemic typhus), Borrelia recurrentis (relapsing fever)

Phormia regina

blowfly

Mechanical N/A

Trypanosoma cruzi (Chagas disease/American trypanosomiasis)

Salmonella (salmonellosis), Streptococcus (pharyngitis etc.)

Salmonella (salmonellosis) 

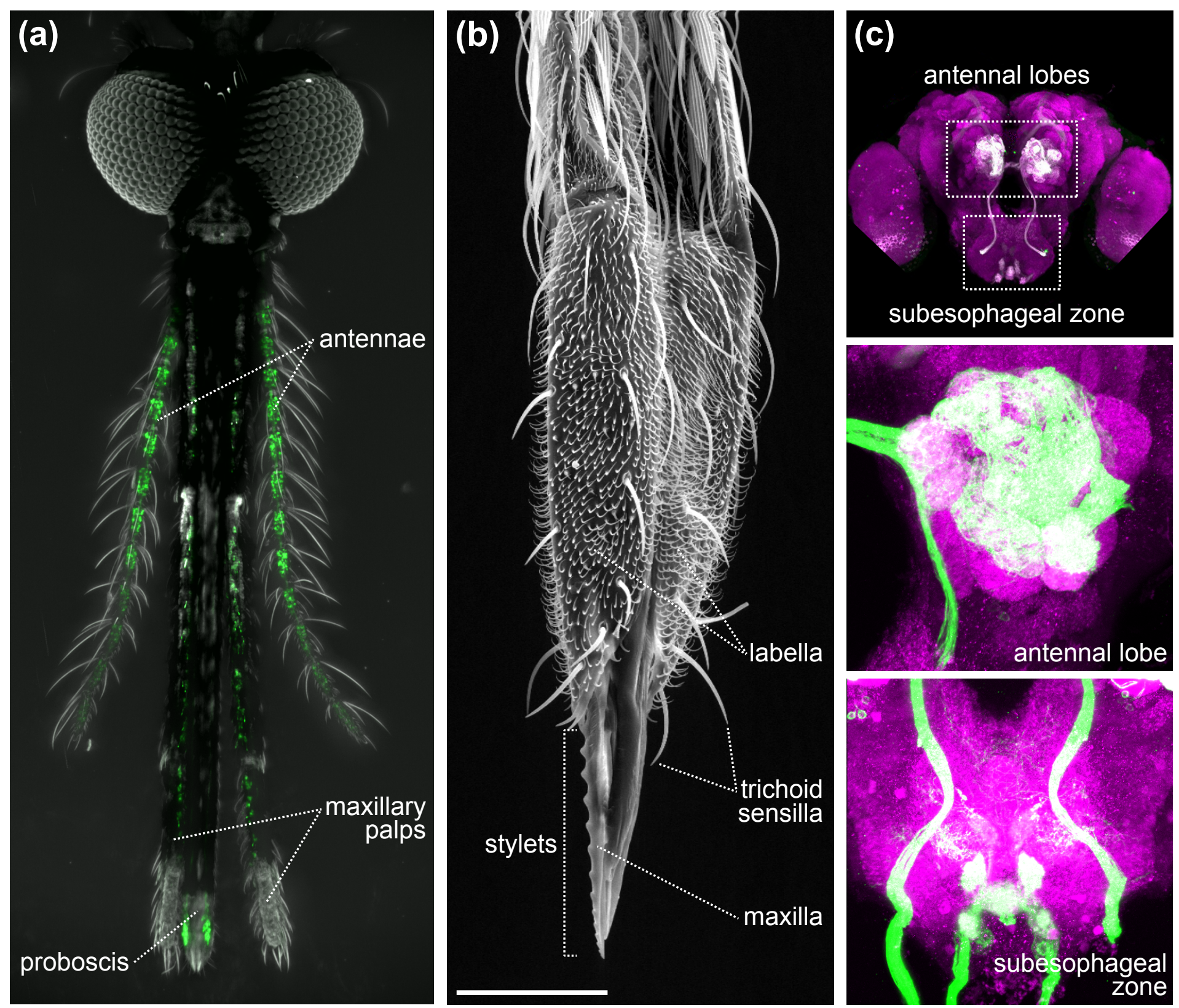